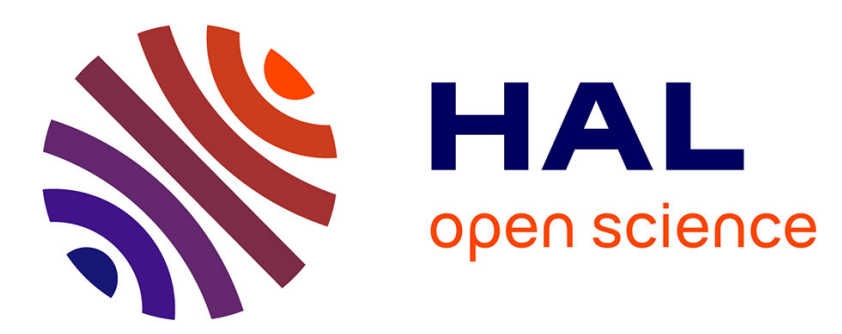

\title{
Parametrization of MIMO multi-controller schemes for bumpless switching
}

\author{
Joseph Julien Yamé, Michel Kinnaert, Thomas Delwiche
}

\section{To cite this version:}

Joseph Julien Yamé, Michel Kinnaert, Thomas Delwiche. Parametrization of MIMO multi-controller schemes for bumpless switching. European Control Conference, ECC'07, Jul 2007, Kos, Greece. pp.CDROM. hal-00164275

\section{HAL Id: hal-00164275 \\ https://hal.science/hal-00164275}

Submitted on 19 Jul 2007

HAL is a multi-disciplinary open access archive for the deposit and dissemination of scientific research documents, whether they are published or not. The documents may come from teaching and research institutions in France or abroad, or from public or private research centers.
L'archive ouverte pluridisciplinaire HAL, est destinée au dépôt et à la diffusion de documents scientifiques de niveau recherche, publiés ou non, émanant des établissements d'enseignement et de recherche français ou étrangers, des laboratoires publics ou privés. 


\title{
Parametrization of MIMO multi-controller schemes for bumpless switching
}

\author{
Joseph Yamé, Michel Kinnaert* and Thomas Delwiche
}

\begin{abstract}
In this paper the realization and implementation of a multi-controller scheme made of a finite set of linear multiple-input-multiple-output controllers, possibly having different state dimensions, is considered. The problem of bumpless switching between the active controller acting in closed-loop and another controller in the set of controllers waiting to take over the control loop is addressed. A non minimal parametrization of the set of controllers such that all controllers share the same state variables is presented, and it is shown that such a parametrization guarantees bumpless switching between any two controllers within the controller set.
\end{abstract}

\section{INTRODUCTION}

In order to deal with changing operating environments, advanced control systems often consist of hybrid dynamical systems which mix continuous-time behavior with a discrete event based decision mechanism. In such control systems, it is common that the output issued by the controller is temporarily different from the input of the controlled process. This difference is caused by a substitution due to the instantaneous switching between two different control laws. Typical instances of input substitution occur in reconfigurable control or multi-controller schemes such as found in flight control for widening aircraft performance envelope, fault-tolerant systems, multi-mode systems, mode switching from manual to automatic industrial controllers, etc... The discrepancy between the process input and the controller output might lead to performance degradation and even instability of the closed-loop system. In order to alleviate this problem, socalled bumpless transfer techniques have been developed.

Bumpless transfer has often been regarded as similar to the anti-windup problem which has attracted a tremendous research activity for many years (see [1], [3], [6], [8] and references therein). In a simple mode switching, where the aim is to switch from manual control to an ultimate controller in the loop, bumpless transfer is usually unidirectional and it is obtained by adding an anti-windup mechanism to the controller to be switched on. In reference [4], the authors pointed out the need to consider bidirectional bumpless transfer between two controllers in modern industrial control

The work of Thomas Delwiche is supported by a FRIA grant

Support by the Interuniversity Attraction Poles Programme, initiated by the Belgian State, Science Policy Office, through the Belgian Network DYSCO and support by "Communauté francaise de Belgique" through "Action de Recherche Concertée" 06/11344 are gratefully acknowledged. The scientific responsibility rests with the authors

Joseph Yamé is with CRAN-UMR 7039 CNRS, Université Henri Poincaré, Nancy I, F-54506 Vandoeuvre-les-Nancy, France

Michel Kinnaert and Thomas Delwiche are with Dept. of Control Engineering and System Analysis, Université Libre de Bruxelles. CP 165/55, 50 Av. F. D. Roosevelt, B-1050 Brussels, Belgium

*Corresponding author email: michel.kinnaert@ulb.ac.be systems where it is often the case that a newly designed controller should be temporarily installed and tested during normal process operation. They derived a bumpless transition scheme by recasting the problem into a tracking problem. In that framework, the output of the standby controller achieves tracking of the online control signal by means of a twodegrees-of-freedom controller. Bidirectionality of the bumpless transfer scheme is obtained by adding a symmetrical tracking loop for the other controller. A direct approach to bumpless transfer in a two-controller configuration is also considered in [9] where an optimal linear quadratic control problem aimed at minimizing the discrepancy between the two controller inputs and outputs is formulated. It results in a feedback gain which acts as a "subcontroller" for the standby controller. Bidirectionality is not considered in that reference, but it should be achievable by symmetry. For multi-controller schemes such as found in hybrid or switched mode systems, multidirectional bumpless transfer should be mandatory, and, to achieve this, the common approach consist in appending an anti-windup mechanism to each controller (see for instance [5] and [11]). In [5], this mechanism consists of high gain feedback loops around all idle controllers, which forces the controller outputs to track the process input, whilst, in [11], each controller is augmented with a dynamics identical to that of the plant in order to allow the controller state to evolve in an appropriate way when the controller is not connected to the plant input. Such bumpless transfer schemes become cumbersome and hard to implement when the number of controllers is large. Besides, in the high gain approach, the unstable zeros of the controllers limit the increase of the gain. Moreover, the technique presented in [11] might fail if a sufficiently accurate model of the plant is not available.

Quite surprisingly in all the above mentioned references the concept of bumpless transfer itself has never been precisely formalized. This gap is addressed in [10] and [12] where the concepts of ideal or target state, input and output responses are introduced. However, the considered bumpless transfer problems are different. In [12], the minimization of the gain from the plant state mismatch (namely the deviation from the ideal state) at the switching instant to the $\mathcal{L}_{2}$-norm of the plant output mismatch is achieved. This requires a bumpless transfer filter involving a model of the plant. In [10] the objective is to find a realization of multi-controller scheme so that the plant state mismatch at the switching instant vanishes in specific conditions. No model of the plant is involved in the latter case. The contributions of the present paper are to further formalize the work of [10] and 
to generalize it to multiple-input-multiple-output controllers.

Notice that the problem of switching between linear timeinvariant (LTI) controllers within a multi-controller scheme, in such a way that the stability of the closed-loop system is assured for any switching sequence, has also been solved by resorting to specific realizations for the controller family in [7]. However, the problem addressed in this paper is different and the resulting realization of the controller family has no link with that of [7]. Indeed, the realization of the latter paper involves the process model, while the realization proposed in this paper exclusively relies on the controller transfer functions. Besides, the equilibrium state of the controller depends of the active controller in the loop in [7], a situation which is avoided here, in order to achieve bumpless switching between the controllers.

The paper is organized as follows. In section II, a discussion and a formal definition of bumpless switching within a multi-controller scheme are given. In section III, a statespace parametrization of the multi-controller is presented and it is proved that it achieves bumpless switching under specific circumstances. In section IV, the proposed controller parametrization is implemented in a simulation in order to illustrate its effectiveness.

\section{Problem Statement}

The linear time invariant process described by the following state space model is considered

$$
\begin{cases}\dot{x}_{P}=A_{P} x_{P}+B_{P} u & x_{P}\left(t_{0}\right)=x_{P 0} \\ y & =C_{P} x_{P}\end{cases}
$$

where $x_{P} \in \mathbb{R}^{n_{x}}$ is the plant state, $u \in \mathbb{R}^{m}$ is the control input and $y \in \mathbb{R}^{p}$ is the measured plant output.

It is controlled by the multi-controller scheme depicted in Figure 1, in which each controller is described as

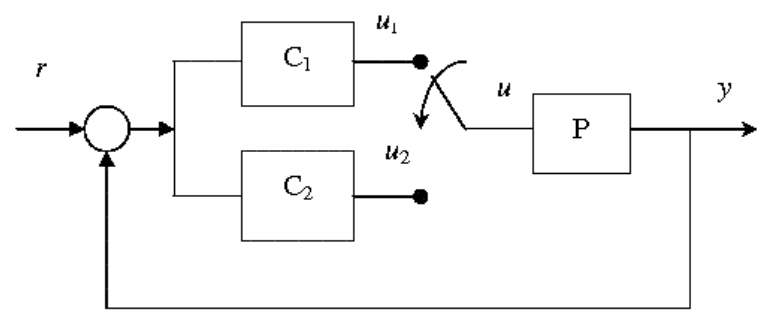

Fig. 1. Switching from controller 1 to controller 2

$$
\left\{\begin{array}{l}
\dot{x}_{i}=F_{i} x_{i}+G_{i}(r-y) ; x_{i}\left(t_{0}\right)=x_{i 0} \\
u_{i}=H_{i} x_{i}+J_{i}(r-y)
\end{array}\right.
$$

where $x_{i}$ denotes the state of the $i^{\text {th }}$ controller $(i=1,2)$ and $r \in \mathbb{R}^{p}$ is the reference signal. It is assumed that both controllers stabilize the process (1), namely the closed-loop systems of Figure 2 (a) and (b) are asymptotically stable.

The control signal $u$ of this switched-mode system is equal either to $u_{1}$ or to $u_{2}$, depending on the position of the switch. In this section, a switching from controller $C_{1}$ to controller

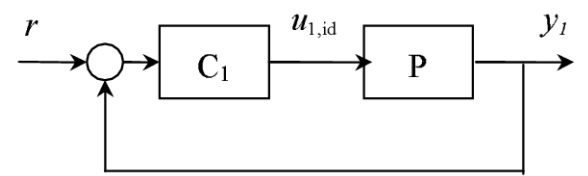

(a)

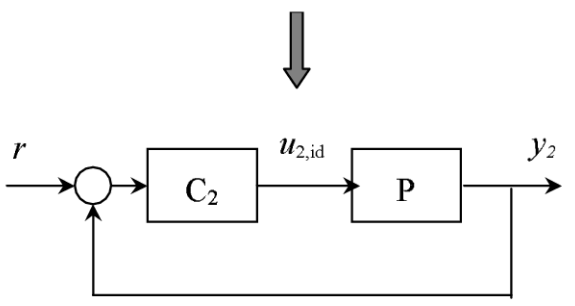

(b)

Fig. 2. Closed-loop systems with (a) controller 1 and (b) controller 2

$C_{2}$ is considered, without loss of generality. It is supposed to occur at an arbitrary time instant $t_{s}>t_{0}$.

The design methodologies for the controllers of such a switched-mode system are usually based on the synthesis of the closed-loop systems composed of the individual controllers as depicted in figure 2, with the desire that the plant output $y$ of the switched-mode system in figure 1 be given by the ideal signal $y_{i d}$ obtained by concatenating $y_{1}$ on $\left[t_{0}, t_{s}\right)$ with signal $y_{2}$ on $\left[t_{s}, \infty\right)$. To define such an ideal or target response uniquely, it is further assumed that, at time $t_{s}$, system (a) and system (b) in figure 2 have reached their steady state response, namely that any effect of initial state has vanished and that the equilibrium input associated to the reference trajectory is applied since $t \rightarrow-\infty$. The corresponding outputs for each loop will be denotes $y_{1, i d}$ and $y_{2, i d}$. Thus $y_{i d}$ is defined as:

$$
y_{i d}(t) \equiv \begin{cases}y_{1, i d}(t) & \text { for } \mathrm{t} \in\left[\mathrm{t}_{0}, \mathrm{t}_{\mathrm{s}}\right) \\ y_{2, i d}(t) & \text { for } \mathrm{t} \geq \mathrm{t}_{\mathrm{s}}\end{cases}
$$

Such a concept of ideal or target response was introduced in [10], [12]. It requires to consider choices of reference signals $r(t), t \in(-\infty, \infty)$ such that the solution of the closed-loop system (1),(2) is unique, both for $i=1$ and $i=2$.

If $P$ denotes the transfer operator of the plant, then the output signal (3) is achieved in the switched-mode system of figure 1 through the following ideal input signal to the plant

$$
u(t)=u_{i d}(t) \equiv \begin{cases}u_{1, i d}(t) & \text { for } \mathrm{t} \in\left[\mathrm{t}_{0}, \mathrm{t}_{\mathrm{s}}\right) \\ u_{2, i d}(t) & \text { for } \mathrm{t} \geq \mathrm{t}_{\mathrm{s}}\end{cases}
$$

where $y_{j, i d}=P u_{j, i d}, j=1,2$. A key feature of the ideal signal $u_{i d}$ is that its segment on $\left[t_{s}, \infty\right)$, i.e. signal $u_{2, i d}$ from $t_{s}$ to $\infty$ is issued from the controller $C_{2}$ which has been constantly connected to the plant in closed-loop. However, due to the very structure of the switched-mode system of figure 1 , the output of controller $C_{2}$ can only be connected to the plant from time $t_{s}$, and consequently its 
state trajectory on $\left[t_{0}, t_{s}\right)$ has no effect on the evolution of the state of the plant in that time interval. The resulting lack of dynamical consistency between the two state trajectories up to the switching instant turns out to be the main cause of the switching transients which impair the performance of the closed-loop system when controller $C_{2}$ becomes active. Bumpless switching should aim at guaranteeing such dynamical consistency.

The actual input signal to the plant of the switched-mode system of figure 1 can be written as a perturbation to the ideal input signal, that is ,

$$
u(t)=u_{i d}(t)+u_{t r}(t) \text { for } \mathrm{t} \in\left[\mathrm{t}_{0}, \infty\right)
$$

where $u_{t r}$ is the transient signal induced by the switching at time $t_{s}$. It is obviously given by $u_{t r}(t)=0$ for $t<t_{s}$ and $u_{t r}(t)=u(t)-u_{i d}(t)$ otherwise. To see how this switching transient arises, let us consider a state space formalism.

The ideal or target response for $t \geq t_{s}$ is the response of the closed-loop system with controller $C_{2}$ constantly in the loop, namely,

$$
\begin{gathered}
\left(\begin{array}{c}
\dot{x}_{2} \\
\dot{x}_{P}
\end{array}\right)=\left(\begin{array}{cc}
F_{2} & -G_{2} C_{P} \\
B_{P} H_{2} & A_{P}-B_{P} J_{2} C_{P}
\end{array}\right)\left(\begin{array}{c}
x_{2} \\
x_{P}
\end{array}\right) \\
+\left(\begin{array}{c}
G_{2} \\
B_{P} J_{2}
\end{array}\right) r \\
y=\left(\begin{array}{ll}
0 & C_{P}
\end{array}\right)\left(\begin{array}{c}
x_{2} \\
x_{P}
\end{array}\right)
\end{gathered}
$$

which is obtained by combining (1) and (2) and substituting $u_{2}$ given by the following expression for $u$ in (1)

$$
u=u_{2}=\left(\begin{array}{cc}
H_{2} & -J_{2} C_{P}
\end{array}\right)\left(\begin{array}{c}
x_{2} \\
x_{P}
\end{array}\right)+J_{2} r
$$

Let $\xi=\left(\begin{array}{ll}x_{2}^{T} & x_{P}^{T}\end{array}\right)^{T}$ denote the state vector of system (6),(7). The equilibrium state trajectory of the closed-loop system with controller $C_{2}$ constantly in the loop, is given by

$$
\xi_{\text {id }}(t)=\int_{-\infty}^{t} \Phi(t, \tau) r(\tau) d \tau \equiv \Theta(t,-\infty) r(t)
$$

where $\Phi(t, \tau)$ is the state transition matrix of system (6), and $\Theta(t,-\infty)$ denotes the integral operator with kernel $\Phi(t, \tau)$. It coïncides with the ideal state trajectory for $t \geq t_{s}$. The ideal control and output signals for $t \geq t_{s}$ are obtained by substituting (9) for the state vector in (8) and (7).

Let us now turn to the scenario where controller $C_{1}$ is in the loop for $t \in\left[t_{0}, t_{s}\right)$ and $C_{2}$ for $t \geq t_{s}$. When controller $C_{1}$ is in the loop, the evolution of the state vectors $x_{2}$ and $x_{P}$ is obtained from (1) and (2) by concatenating the state of the process and the multi-controller and imposing that $u=u_{1}$, which yields:

$$
\left(\begin{array}{c}
\dot{x}_{2} \\
\dot{x}_{P} \\
\dot{x}_{1}
\end{array}\right)=\left(\begin{array}{ccc}
F_{2} & -G_{2} C_{P} & 0 \\
0 & A_{P}-B_{P} J_{1} C_{P} & B_{P} H_{1} \\
0 & -G_{1} C_{P} & F_{1}
\end{array}\right)\left(\begin{array}{c}
x_{2} \\
x_{P} \\
x_{1}
\end{array}\right)
$$

$$
+\left(\begin{array}{c}
G_{2} \\
B_{P} J_{1} \\
G_{1}
\end{array}\right) r
$$

Indeed, setting $\eta=\left(\begin{array}{ll}\xi^{T} & x_{1}^{T}\end{array}\right)^{T}$ and solving (10) for the initial condition $\eta\left(t_{0}\right)=\left(\xi_{0}^{T}, x_{10}^{T}\right)^{T}$ yields the state trajectory

$$
\eta(t)=\eta\left(t ; t_{0}, \eta_{0}, r\right) \quad t \in\left[t_{0}, t_{s}\right)
$$

from which the trajectory of $\xi$ for $t \in\left[t_{0}, t_{s}\right)$ when controller $C_{1}$ is in the loop can be extracted. This yields $\xi\left(t_{s}^{-}\right)$as the left limit of $\xi(t)$ at $t=t_{s}$.

Now, for $t \geq t_{s}$, controller $C_{2}$ stands in the loop, and the evolution of $\xi$ is obtained from (6) with initial condition $\xi\left(t_{s}^{-}\right)$at time $t_{s}$, namely

$$
\xi(t)=\Phi\left(t, t_{s}\right) \xi\left(t_{s}^{-}\right)+\Theta\left(t, t_{s}\right) r(t) \quad t \geq t_{s}
$$

This is to be compared with the evolution of $\xi$ when controller $C_{2}$ is constantly connected to the plant

$$
\xi_{i d}(t)=\Phi\left(t, t_{s}\right) \xi_{i d}\left(t_{s}\right)+\Theta\left(t, t_{s}\right) r(t) \quad t \geq t_{s}
$$

The switching transient is thus the free motion given by the difference $\xi_{t r}(t)$ between trajectories (12) and (13), that is

$$
\xi_{\text {tr }}(t)=\Phi\left(t, t_{s}\right)(\Delta \xi)_{t_{s}} \quad t \geq t_{s}
$$

where $(\Delta \xi)_{t_{s}}=\xi_{i d}\left(t_{s}\right)-\xi\left(t_{s}^{-}\right)$, and the associated transient in the input signal to the plant is directly deduced from (8), namely

$$
u_{t r}(t)=\left(\begin{array}{cc}
H_{2} & -J_{2} C_{P}
\end{array}\right) \xi_{t r}(t)
$$

It is now clear that no switching transient will occur if $(\Delta \xi)_{t_{s}}$, the mismatch between the ideal and the actual state vector $\xi$ at time $t_{s}$ is zero. This holds true, even if the input experiences a jump $(\Delta u)_{t_{s}}=u_{2}\left(t_{s}^{+}\right)-u_{1}\left(t_{s}^{-}\right) \neq 0$ at the switching instant (the arguments $t_{s}^{+}$and $t_{s}^{-}$stand respectively for the right and left limit at $t_{s}$ of the corresponding signal). The switching transient results from an inconsistency between the state of the plant $x_{P}$ and the state of the controller $x_{2}$. Consistency is assured when the controller $C_{2}$ is constantly connected to the plant and it is characterized by vector $\xi_{i d}(t)$, in which case $x_{P}$ depends on $x_{2}$ (see (6)). When $C_{1}$ is in the loop however, the plant state evolves independently from the state of the idle controller, as can be seen from the zeroed $(2,1)$ entry of the state matrix in equation (10). Since in a switched-mode system, only one controller can be effectively connected to the plant at each time instant, a solution to the bumpless switching problem inspired from the above analysis is to realize a virtual constant connection of all idle controllers to the plant so as to assure the consistency between the state of the plant and the states of all controllers. This approach is the object of the next section.

Based on the above discussion, a formal problem statement for bumpless switching in a switched-mode control system can be stated as follows.

Problem statement: Bumpless switching 


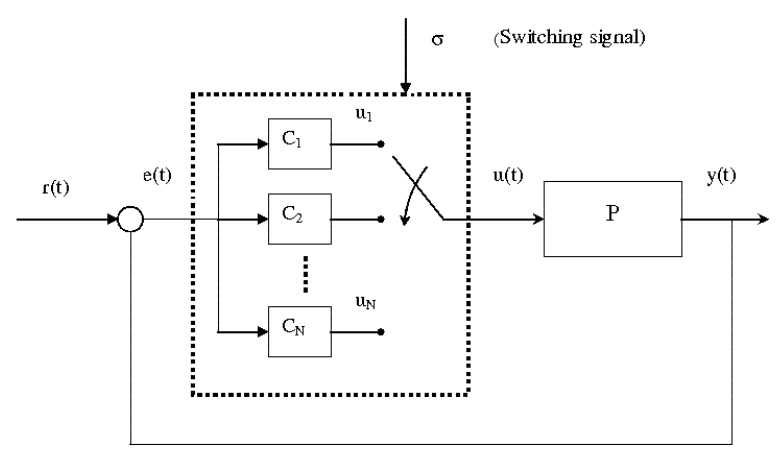

Fig. 3. Switched-mode control system

Consider plant (1) and the set of controllers (2), with $i=$ $1, \cdots, N$, corresponding to the switched-mode control system of figure 3. Assume that each of the controllers stabilizes the plant and that there is a unique equilibrium state trajectory associated to the reference trajectory $r(t), t \in(-\infty, \infty)$ for each of the $N$ controllers. This scheme achieves bumpless switching if the following statement holds for any pair of controllers say $(j, k), j \neq k, j, k \in\{1, \cdots, N\}$ :

Consider an arbitrary time instant $t_{s}$ at which the closedloop system with controller $j$ as the active controller has reached the equilibrium state trajectory. Upon switching to controller $k$ at time instant $t_{s}$, the state trajectory of the closed-loop system coïncides, for $t \geq t_{s}$, with the equilibrium state trajectory for the closed-loop system with the active controller $k$.

In the next section, a shared-state parametrization of a switched-mode controller is presented and the resulting switched-mode control system is shown to achieve bumpless switching.

\section{PARAMETRIZATION}

Let us rewrite the $N$ control laws in terms of transfer matrices:

$$
u_{i}(s)=C_{i}(s) e(s)=\frac{\tilde{B}_{i}(s)}{\tilde{a}_{i}(s)} e(s) \quad i=1, \cdots, N
$$

where $e(s)$ stands for the control error $r(s)-y(s), C_{i}(s)$ is a $m \times p$ transfer matrix, $\tilde{B}_{i}(s)$ is a $m \times p$ polynomial matrix and $\tilde{a}_{i}(s)$ is a monic polynomial constructed as the smallest common multiple of all the denominators of the elements of $C_{i}(s)$. Set $n=\max _{i=1, N} \operatorname{deg}\left(\tilde{a}_{i}(s)\right)$ where $\operatorname{deg}\left(\tilde{a}_{i}(s)\right)$ denotes the degree of polynomial $\tilde{a}_{i}(s)$. Then all transfer matrices $C_{i}(s)$ can always be written in the form

$$
C_{i}(s)=\frac{B_{i}(s)}{a_{i}(s)}
$$

where the denominator polynomials $a_{i}(s), i=1, \cdots, N$ are monic polynomials of degree $n$, namely

$$
a_{i}(s)=s^{n}+a_{i, 1} s^{n-1}+\cdots+a_{i, n-1} s+a_{i, n}
$$

and the numerator polynomial matrix can be written

$$
B_{i}(s)=B_{i, 0} s^{n}+B_{i, 1} s^{n-1}+\cdots+B_{i, n} .
$$

All additional roots of $a_{i}(s)$ with respect to the roots of $\tilde{a}_{i}$ should be placed in the open-left half plane. These axtra roots are needed to obtain a shared-state parametrization for all controllers, as will be seen below. They do not play any role on the input-output dynamics of the closed-loop system.

Each controller transfer matrix can be separated in a direct feedthrough part $B_{i, 0}$ and a strictly proper part $C_{i}^{\prime}(s)$ :

$$
C_{i}(s)=C_{i}^{\prime}(s)+B_{i, 0}
$$

The $i^{\text {th }}$ controller output can be written as

$$
u_{i}(s)=u_{i}^{\prime}(s)+B_{i, 0} e(s)
$$

where $u_{i}^{\prime}(s)$ is defined by

$$
\begin{aligned}
u_{i}^{\prime}(s) & =\frac{B_{i}(s)-B_{i, 0} a_{i}(s)}{a_{i}(s)} e(s)=\frac{B_{i}^{\prime}(s)}{a_{i}(s)} e(s) \\
& =\frac{B_{i, 1}^{\prime} s^{n-1}+\cdots+B_{i, n}^{\prime}}{s^{n}+a_{i, 1} s^{n-1}+\cdots+a_{i, n-1} s+a_{i, n}} e(s)
\end{aligned}
$$

Let $\lambda(s)$ be a Hurwitz monic polynomial of order $n$ such that $\lambda(s) \neq a_{i}(s), i=1, \cdots, N$. Then (18) can be rewritten in a feedback form (see Figure 4) as follows

$$
u_{i}^{\prime}(s)=\frac{B_{i}^{\prime}(s)}{\lambda(s)} e(s)+\frac{\lambda(s)-a_{i}(s)}{\lambda(s)} u_{i}^{\prime}(s)
$$

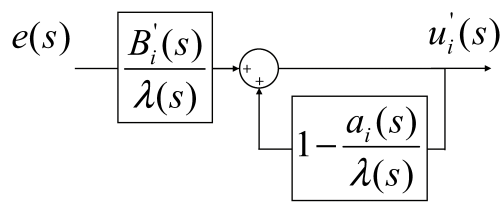

Fig. 4. Feedback loop described by (19)

For notation convenience, define

$$
\begin{aligned}
d_{i}(s)= & \lambda(s)-a_{i}(s) \\
= & \left(\lambda_{1}-a_{i, 1}\right) s^{n-1}+\left(\lambda_{2}-a_{i, 2}\right) s^{n-2} \\
& +\cdots+\left(\lambda_{n}-a_{i, n}\right) \\
= & d_{i, 1} s^{n-1}+\cdots+d_{i, n}
\end{aligned}
$$

and introduce the constant matrices

$$
\mathcal{E}_{i}=\left(\begin{array}{ll}
B_{i, n}^{\prime} & B_{i, n-1}^{\prime} \\
\cdots & B_{i, 1}^{\prime}
\end{array}\right)
$$

and

$$
\mathcal{D}_{i}=\left(\begin{array}{lll}
d_{i, n} I_{m} & d_{i, n-1} I_{m} \cdots d_{i, 1} I_{m}
\end{array}\right) .
$$

Equation (19) can be expressed as:

$$
\begin{aligned}
& u_{i}^{\prime}(s)=\left(\begin{array}{ll}
B_{i, n}^{\prime} & B_{i, n-1}^{\prime} \cdots B_{i, 1}^{\prime}
\end{array}\right)\left(\begin{array}{l}
I_{p} \\
s I_{p} \\
\vdots \\
s^{n-1} I_{p}
\end{array}\right) \frac{1}{\lambda(s)} e(s) \\
& +\left(\begin{array}{ll}
d_{i, n} I_{m} & \left.d_{i, n-1} I_{m} \cdots d_{i, 1} I_{m}\right) \\
s I_{m} \\
\vdots \\
s^{n-1} I_{m}
\end{array}\right) \frac{1}{\lambda(s)} u_{i}^{\prime}(s) \\
& =\mathcal{E}_{i} \mathcal{F}_{p}(s) e(s)+\mathcal{D}_{i} \mathcal{F}_{m}(s) u_{i}^{\prime}(s)
\end{aligned}
$$


where $I_{m}$ is the $m \times m$ identity matrix,

$$
\mathcal{F}_{m}(s)=\left(\begin{array}{l}
I_{m} \\
s I_{m} \\
\vdots \\
s^{n-1} I_{m}
\end{array}\right) \frac{1}{\lambda(s)}
$$

and a similar definition holds for $\mathcal{F}_{p}(s)$.

It is straightforward to prove (see e.g. [2]) that the transfer matrix $\mathcal{F}_{m}(s)$ can be expressed in state space form as

$$
\mathcal{F}_{m}(s)=\left(s I-\mathcal{A}_{m}\right)^{-1} \mathcal{B}_{m}
$$

where $\mathcal{A}_{m}$ is in the following block companion form

$$
\mathcal{A}_{m}=\left(\begin{array}{cccccc}
0 & I_{m} & 0 & \cdots & \cdots & 0 \\
0 & 0 & I_{m} & 0 & \cdots & 0 \\
\vdots & \vdots & & \ddots & \ddots & \vdots \\
0 & 0 & \ldots & \cdots & 0 & I_{m} \\
-\lambda_{n} I_{m} & -\lambda_{n-1} I_{m} & \cdots & \cdots & \cdots & -\lambda_{1} I_{m}
\end{array}\right)
$$

and

$$
\mathcal{B}_{m}=\left(\begin{array}{c}
0 \\
\vdots \\
0 \\
I_{m}
\end{array}\right)
$$

Introducing (20) into (17) yields the following expression for the $i^{t h}$ control law:

$$
u_{i}(s)=\mathcal{E}_{i} \mathcal{F}_{p}(s) e(s)+\mathcal{D}_{i} \mathcal{F}_{m}(s) u_{i}^{\prime}(s)+B_{i, 0} e(s)
$$

Now, let us define the state variables $\zeta_{1}(s)=\mathcal{F}_{p}(s) e(s)$ and $\zeta_{2}(s)=\mathcal{F}_{m}(s) u_{i}^{\prime}(s)$. By resorting to (22) a state space representation of the control law (23) is obtained:

$$
\begin{aligned}
\left(\begin{array}{c}
\dot{\zeta}_{1}(t) \\
\dot{\zeta}_{2}(t)
\end{array}\right)= & \left(\begin{array}{cc}
\mathcal{A}_{p} & 0 \\
0 & \mathcal{A}_{m}
\end{array}\right)\left(\begin{array}{l}
\zeta_{1}(t) \\
\zeta_{2}(t)
\end{array}\right) \\
& +\left(\begin{array}{cc}
\mathcal{B}_{p} & 0 \\
0 & \mathcal{B}_{m}
\end{array}\right)\left(\begin{array}{c}
e(t) \\
u_{i}^{\prime}(t)
\end{array}\right) \\
u_{i}(t)= & \mathcal{E}_{i} \zeta_{1}(t)+\mathcal{D}_{i} \zeta_{2}(t)+B_{i, 0} e(t)
\end{aligned}
$$

The desired parametrization is obtained by substituting $u_{i}(t)-B_{i, 0} e(t)$ for $u_{i}^{\prime}(t)$ in the state equation, and then substituting $u(t)$ for $u_{i}(t)$. The latter substitution does not change the control law if the $i^{t h}$ controller is the active controller $(\sigma(t)=i)$. However, if this is not the case, it modifies the controller state in such a way that it only depends on the active controller at each time instant. This is actually one of the keys to achieve bumpless switching. The final parametrization for the $i^{\text {th }}$ controller is described by

$$
\begin{aligned}
\left(\begin{array}{l}
\dot{\zeta}_{1}(t) \\
\dot{\zeta}_{2}(t)
\end{array}\right)= & \left(\begin{array}{cc}
\mathcal{A}_{p} & 0 \\
0 & \mathcal{A}_{m}
\end{array}\right)\left(\begin{array}{l}
\zeta_{1}(t) \\
\zeta_{2}(t)
\end{array}\right) \\
& +\left(\begin{array}{cc}
\mathcal{B}_{p} & 0 \\
-\mathcal{B}_{m} B_{0, \sigma(t)} & \mathcal{B}_{m}
\end{array}\right)\left(\begin{array}{c}
e(t) \\
u(t)
\end{array}\right) \\
u_{i}(t)= & \mathcal{E}_{i} \zeta_{1}(t)+\mathcal{D}_{i} \zeta_{2}(t)+B_{i, 0} e(t)
\end{aligned}
$$

where $\sigma(t)$ indicates the controller which is presently active in the loop.
Notice that the multi-controller itself can now be viewed as a system with input $e$ and output $u$ described by

$$
\left\{\begin{array}{l}
\dot{\zeta}(t)=\mathcal{A} \zeta(t)+\mathcal{B}_{u} u(t)+\mathcal{B}_{e} e(t) \\
u_{i}(t)=\mathcal{C}_{i} \zeta(t)+B_{i, 0} e(t) \\
u(t)=u_{\sigma(t)}(t)
\end{array} i=1,2, \cdots, N\right.
$$

where $\zeta(t)=\left(\begin{array}{ll}\zeta_{1}^{T}(t) & \zeta_{2}^{T}(t)\end{array}\right)^{T}$ and

$$
\begin{array}{cc}
\mathcal{A}=\left(\begin{array}{cc}
\mathcal{A}_{p} & 0 \\
0 & \mathcal{A}_{m}
\end{array}\right) & \mathcal{B}_{e}=\left(\begin{array}{c}
\mathcal{B}_{p} \\
-\mathcal{B}_{m} B_{0, \sigma(t)}
\end{array}\right) \\
\mathcal{B}_{u}=\left(\begin{array}{c}
0 \\
\mathcal{B}_{m}
\end{array}\right) & \mathcal{C}_{i}=\left(\begin{array}{ll}
\mathcal{E}_{i} & \mathcal{D}_{i}
\end{array}\right)
\end{array}
$$

The main property of this parametrization is summarized in the following theorem.

Theorem 1: Consider the switched-mode system of figure 3. If

1) each controller is a stabilizing controller for plant (1),

2) perfect tracking of the reference is achieved asymptotically for each controller,

3) for the given plant, there is a unique control trajectory associated to the reference trajectory at equilibrium,

then the parametrization (28) achieves bumpless switching.

Proof 1: Let us consider an arbitrary controller pair $(j, k), j \neq k, j, k \in\{1,2, \cdots N\}$. Our aim is to show that, starting from an equilibrium situation in which controller $j$ is in the loop, upon switching to controller $k$ the closed-loop system instantaneously follows the ideal trajectory associated to that controller. Given hypothesis 2, the ideal (equilibrium) trajectory of the plant output coïncides with the reference signal $r$ which yields a zero tracking error $e$. The equilibrium state and control signal with controller $\mathrm{k}$ in the loop thus fulfill

$$
\begin{aligned}
\dot{\zeta}_{i d} & =\mathcal{A} \zeta_{i d}+\mathcal{B}_{u} u_{i d} \\
u_{i d} & =\mathcal{C}_{k} \zeta_{i d} \\
\dot{x}_{P, i d} & =A_{P} x_{P, i d}+B_{P} u_{i d} \\
r & =C_{P} x_{P, i d}
\end{aligned}
$$

Due to hypothesis $3, u_{i d}$ is uniquely defined by the last two equations. This input trajectory is associated to a unique trajectory for the state of the controller at equilibrium obtained by solving (29):

$$
\zeta_{i d}(t)=\int_{-\infty}^{t} e^{\mathcal{A}(t-\tau)} \mathcal{B}_{u} u_{i d}(\tau) d \tau
$$

This state trajectory is independent of the particular controller which lies in the loop, since $\mathcal{A}$ and $\mathcal{B}_{u}$ are common to all controllers. Therefore $\zeta_{i d}(t)$ is also the state trajectory reached at equilibrium when any controller, and in particular the $j^{t h}$ 's one, lies in the closed-loop. Since all controllers reach the same ideal state at equilibrium, bumpless switching is achieved.

Remark 1: Hypothesis 2 can be fulfilled by resorting to the internal model principle in the design of each controller

Remark 2: A common particular case is the tracking of a piece-wise constant reference signal for a square $(m=p)$ 
process with a non-singular steady state gain. In this situation the ideal process input is directly computed from

$$
u_{i d}=P(0)^{-1} r
$$

and the ideal state trajectory for the controller results from (29) by setting the derivative to zero

$$
\zeta_{\text {id }}=-\mathcal{A}^{-1} \mathcal{B}_{u} u_{i d}
$$

Remark 3: The parametrization of the set of controllers obtained in continuous time extends mutatis mutandis to the discrete time setting, and the statement of the bumpless switching problem remains valid in that setting.

\section{EXAMPLE}

As a simple example illustrating the bumpless switching property of the proposed parametrization for a multicontroller scheme, consider a fluid mixing plant depicted in figure 5. This plant is made of three tanks. A first one contains colored water (coloring concentration denoted by $C_{c}(t)$ ) and a second one contains clear water. These two tanks are connected to a third one (the mixer) where the mixing of the clear and colored water takes place. The aim is to achieve a specific level $h(t)$ and concentration $C_{o}(t)$ in the mixer by acting on the flows of colored and clear water (denoted by $Q_{1}(t)$ and $Q_{2}(t)$ respectively). Mixing is supposed to be perfect and to take place instantaneously. Besides, the output flow $Q_{o}(t)$ and input concentration $C_{c}(t)$ are assumed to be fixed at known nominal values $Q_{o}^{*}$ and $C_{c}^{*}$. Here, the asterisk indicates values corresponding to the nominal operating point, for which the numerical values are given in table I. A state-space model of the mixer is easily deduced from material conservation laws. Linearizing the resulting equations around the indicated operating point yields expressions (34) and (35) where $S$ denotes the section of the cylindrical mixer (equal to $0.01 \mathrm{~m}^{2}$ ). By an abuse of notation, the same symbols are used to denote the original variables and their deviation with respect to the operating point.

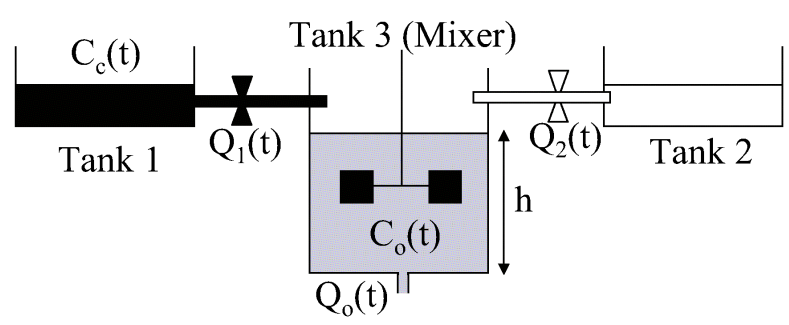

Fig. 5. Fluid mixing plant.

$$
\begin{aligned}
\left(\begin{array}{c}
\dot{h}(t) \\
\dot{C}_{o}(t)
\end{array}\right) & =\left(\begin{array}{cc}
0 & 0 \\
0 & -\frac{Q_{o}^{*}}{S h^{*}}
\end{array}\right)\left(\begin{array}{c}
h(t) \\
C_{o}(t)
\end{array}\right) \\
& +\left(\begin{array}{cc}
\frac{C_{c}^{*}-C_{o}^{*}}{S h^{*}} & \frac{-C_{o}^{*}}{S h^{*}}
\end{array}\right)\left(\begin{array}{c}
Q_{1}(t) \\
Q_{2}(t)
\end{array}\right)
\end{aligned}
$$

\begin{tabular}{|c|c|}
\hline$Q_{o}^{*}$ & $0.015 \mathrm{l} / \mathrm{s}$ \\
\hline$h^{*}$ & $0.15 \mathrm{~m}$ \\
\hline$Q_{1}^{*}$ & $0.0075 \mathrm{l} / \mathrm{s}$ \\
\hline$Q_{2}^{*}$ & $0.0075 \mathrm{l} / \mathrm{s}$ \\
\hline$C_{c}^{*}$ & 0.3 \\
\hline$C_{o}^{*}$ & 0.15 \\
\hline
\end{tabular}

TABLE I

NUMERICAL VALUES ASSOCIATED TO THE OPERATING POINT.

$$
\left(\begin{array}{c}
h(t) \\
C_{o}(t)
\end{array}\right)=\left(\begin{array}{ll}
1 & 0 \\
0 & 1
\end{array}\right)\left(\begin{array}{c}
h(t) \\
C_{o}(t)
\end{array}\right)
$$

Two decoupling controllers were designed for this system. They are characterized by the following discrete transfer matrices (sampling period $0.02 \mathrm{~s}$ )

$$
\begin{gathered}
C_{1}(z)=\left(\begin{array}{cc}
0.005 & \frac{0.0025 z-0.0024995}{z-1} \\
0.005 & \frac{-0.0025 z+0.0024995}{z-1}
\end{array}\right) \\
C_{2}(z)=\left(\begin{array}{cc}
\frac{0.019595 z-0.019205}{z-1} & \frac{0.00275 z-0.00225}{z-1} \\
\frac{0.019595 z-0.019205}{z-1} & \frac{-0.00275 z+0.00225}{z-1}
\end{array}\right)
\end{gathered}
$$

After parametrization, the state equations of these two controllers are given in discrete time by expressions (38) if controller $C_{1}$ is connected in the loop and (39) if controller $C_{2}$ is connected in the loop. The output of controllers $C_{1}$ and $C_{2}$ are given respectively by expressions (40) and (41).

$$
\begin{aligned}
& \left(\begin{array}{c}
\zeta_{1}(k+1) \\
\zeta_{2}(k+1)
\end{array}\right)=\left(\begin{array}{cccc}
0.5 & 0 & 0 & 0 \\
0 & 0.5 & 0 & 0 \\
0 & 0 & 0.5 & 0 \\
0 & 0 & 0 & 0.5
\end{array}\right)\left(\begin{array}{l}
\zeta_{1}(k) \\
\zeta_{2}(k)
\end{array}\right) \\
& +\left(\begin{array}{cccc}
1 & 0 & 0 & 0 \\
0 & 1 & 0 & 0 \\
-0.005 & -0.0025 & 1 & 0 \\
-0.005 & 0.0025 & 0 & 1
\end{array}\right)\left(\begin{array}{l}
e(k) \\
u(k)
\end{array}\right) \\
& \left(\begin{array}{c}
\zeta_{1}(k+1) \\
\zeta_{2}(k+1)
\end{array}\right)=\left(\begin{array}{cccc}
0.5 & 0 & 0 & 0 \\
0 & 0.5 & 0 & 0 \\
0 & 0 & 0.5 & 0 \\
0 & 0 & 0 & 0.5
\end{array}\right)\left(\begin{array}{l}
\zeta_{1}(k) \\
\zeta_{2}(k)
\end{array}\right) \\
& +\left(\begin{array}{cccc}
1 & 0 & 0 & 0 \\
0 & 1 & 0 & 0 \\
-0.019595 & -0.00275 & 1 & 0 \\
-0.019595 & 0.00275 & 0 & 1
\end{array}\right)\left(\begin{array}{c}
e(k) \\
u(k)
\end{array}\right) \\
& u_{1}(k)=\left(\begin{array}{cc}
0 & 0.0000005 \\
0 & -0.0000005
\end{array}\right) \zeta_{1}(k)+\left(\begin{array}{cc}
0.5 & 0 \\
0 & 0.5
\end{array}\right) \zeta_{2}(k) \\
& +\left(\begin{array}{cc}
0.005 & 0.0025 \\
0.005 & -0.0025
\end{array}\right) e(k)
\end{aligned}
$$



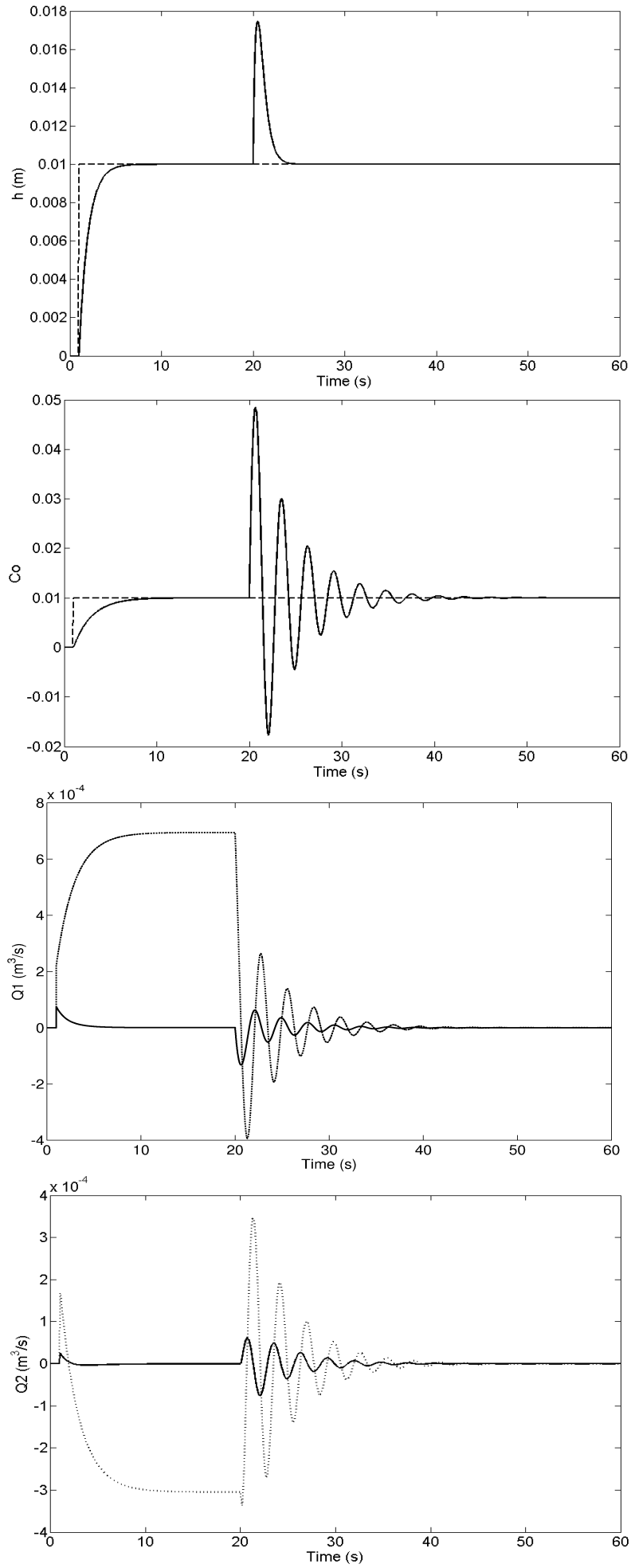

Fig. 6. Inputs and outputs of the plant without parametrization. In the first two figures of this column, the interrupted line represents the reference step. In the remaining figures, the full line represents the output of $C_{1}$ and the dotted line the output of $C_{2}$.
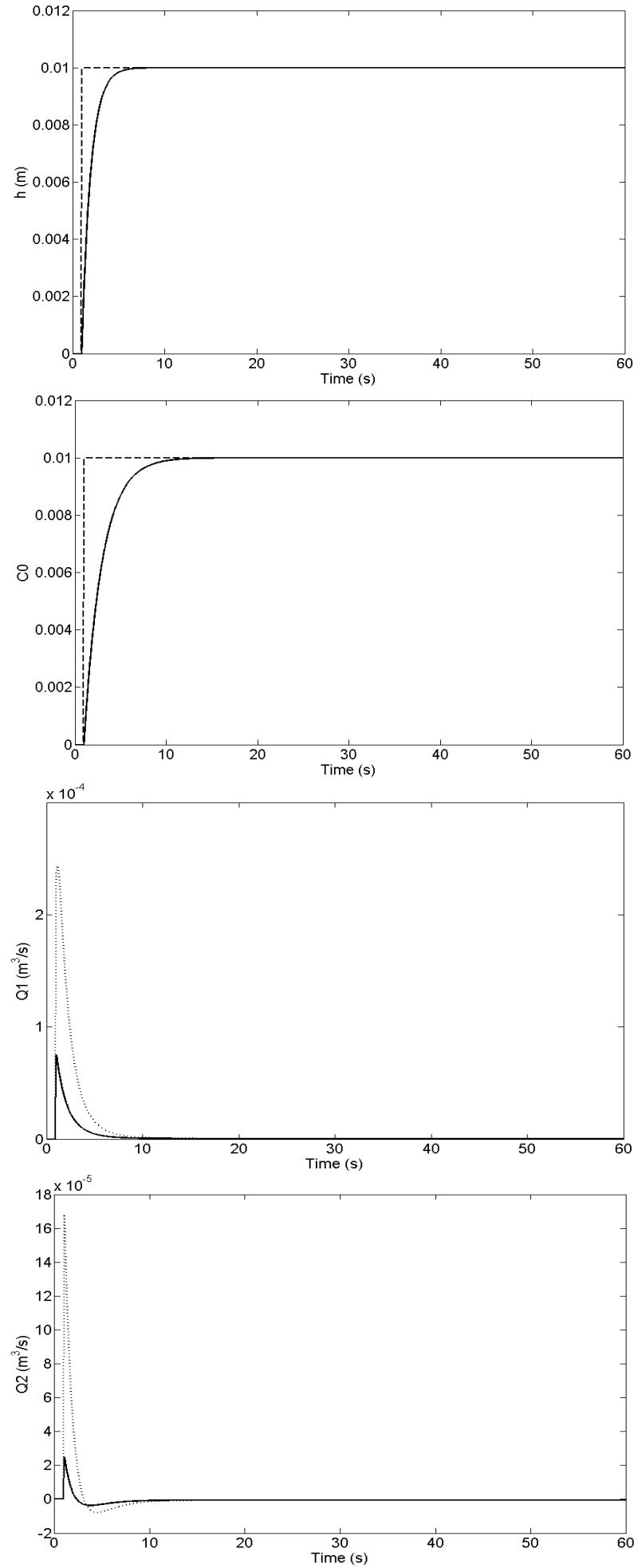

Fig. 7. Inputs and outputs of the plant with parametrization. In the first two figures of this column, the interrupted line represents the reference step. In the remaining figures, the full line represents the output of $C_{1}$ and the dotted line the output of $C_{2}$. 


$$
\begin{aligned}
u_{2}(k)= & \left(\begin{array}{cc}
0.00039 & 0.0005 \\
0.00039 & -0.0005
\end{array}\right) \zeta_{1}(k)+\left(\begin{array}{cc}
0.5 & 0 \\
0 & 0.5
\end{array}\right) \zeta_{2}(k) \\
& +\left(\begin{array}{cc}
0.019595 & 0.00275 \\
0.019595 & -0.00275
\end{array}\right) e(k)
\end{aligned}
$$

Figures 6 and 7 show the process inputs and outputs during a simulation. The reference is initially equal to zero. At $t=1 \mathrm{~s}$, a reference step of magnitude 0.01 is imposed to both outputs. First, controller $C_{1}$ is in the loop, then at $t=20 \mathrm{~s}$, it is replaced by controller $C_{2}\left(u(t)=u_{1}(t)\right.$ for $t<20 \mathrm{~s}$ and $u(t)=u_{2}(t)$ for $\left.t \geq 20 \mathrm{~s}\right)$. Note that without parametrization (figure 6), the control system experiences an undesirable transient which significantly deteriorates the tracking performance after switching. With the proposed controller parametrization (figure 7), the multi-controller shows successful bumpless switching without any transients after switching and it is worth noticing how the pending controller output tracks the actual process input.

\section{CONCLUSions}

In this paper, bumpless switching in the case of multicontrollers schemes was achieved by using a specific statespace parametrization of the set of controllers.

First, bumpless switching was defined formally in terms of a given response of the system. This response corresponds to the ideal response that would be obtained if all controllers were physically and constantly connected to the plant in closed-loop, providing dynamical consistency between the state trajectory of the controllers and the state trajectory of the plant. Based on this analysis, a solution to the bumpless switching appeared to be a constant virtual connection between all idle controllers and the physical plant.

Next, a state-space parametrization of the set of controller assuring this virtual connection was obtained. It was demonstrated that this parametrization achieves bumpless switching under the following hypothesis:

1) each controller is a stabilizing controller for the plant

2) perfect tracking of reference is achieved asymptotically for each controller

3) for the given plant, there is a unique control trajectory associated to the reference trajectory at equilibrium

Finally, an example was presented in order to illustrate the effectiveness of the proposed method for bumpless switching.

\section{REFERENCES}

[1] K.J. Aström and B. Wittenmark. Computer -Controlled Systems: Theory and Design, Prentice-Hall, Engelwood Cliffs, New Jersey, 1984.

[2] C-T. Chen. Linear System Theory and Design, Sauders College Publishing, New York, 1984.

[3] A.H. Glattfelder et al. (Editors). Anti-Windup Control, European Journal of Control, Special Issue, vol. 6, no. 5, 2000.

[4] S.F. Graebe and A.L.B. Ahlén. Dynamic Transfer Among Alternative Controllers and Its relation to Anti-windup Controller design, IEEE Transactions on Control Systems Technology, vol.4, no.1, pp.92-99, 1996.

[5] M. Green and D.J.N. Limebeer. Linear Robust Control Prentice-Hall Inc., Engelwood Cliffs, New Jersey, 1995.
[6] R. Hanus, M. Kinnaert and J.L. Henrotte. Conditionning Technique, a General Anti-Windup and Bumpless Transfer Method, Automatica, vol. 23 , no. 6 , pp. $729-739$.

[7] J.P. Hespanha and A.S. Morse. Switching between stabilizing controllers, Automatica, 38, pp 1905-1917, 2002.

[8] Y. Peng, D. Vrancic and R. Hanus. Anti-Windup, Bumpless and Conditioned Transfer Techniques for PID Controllers, IEEE Control Systems Magazine, vol. 10, no. 4, pp. 48-57, 1996.

[9] M.C. Turner and D.J. Walker. Linear Quadratic Bumpless Transfer, Automatica, vol. 36, pp. 1089-1101, 2000.

[10] J. Yamé and M. Kinnaert. Parametrization of Linear Controllers for Bumpless Switching in Multi-Controller Schemes, AIAA Guidance, Navigation, and Control Conference and Exhibit, Providence, Rhode Island, 2004.

[11] L. Zaccarian and A.R. Teel. A Common Framework for Anti-Windup, Bumpless Transfer and Reliable Designs, Automatica, vol. 38, pp. 1734-1744, 2002.

[12] L. Zaccarian and A.R. Teel. The $\mathcal{L}_{2}\left(l_{2}\right)$ bumpless transfer problem for linear plants: Its definition and solution, Automatica, vol. 41, pp. 1273-1280, 2005. 